\title{
Influence of the Power Generation Capacity of the Structural Parameters of a Piezoelectric Bimorph
}

\author{
Xiaochao Tian $\mathbb{D}^{1,2}$, Jinzhi Zhu $\mathbb{D}^{1,2}$ Zhicong Wang $\mathbb{D}^{1,2}$ Hu Wang $\mathbb{D}^{1,2}$ Yuze Sun $\mathbb{D}^{1,2}$ \\ HaiGang Wang $\mathbb{D},{ }^{1,2}$ Sida Zhang $\mathbb{D}{ }^{3}$ and Zhigang Yang $\mathbb{D}^{3}$ \\ ${ }^{1}$ Institute of Mechanical and Vehicle Engineering, Changchun University, Changchun, China \\ ${ }^{2}$ Jilin Provincial Key Laboratory of Human Health Status Identification and Function Enhancement, Changchun, China \\ ${ }^{3}$ College of Mechanical and Aerospace Engineering, Jilin University, Changchun, China
}

Correspondence should be addressed to Xiaochao Tian; tianxczb@163.com

Received 15 May 2021; Revised 30 June 2021; Accepted 7 July 2021; Published 19 July 2021

Academic Editor: Jiang Jin

Copyright ( 92021 Xiaochao Tian et al. This is an open access article distributed under the Creative Commons Attribution License, which permits unrestricted use, distribution, and reproduction in any medium, provided the original work is properly cited.

With the popularization of integrated circuits, MEMS, and portable electronic devices, chemical batteries have many disadvantages as the main energy supply method, such as large size, high quality, and limited energy supply life, requiring regular replacement, resulting in waste of materials, environmental pollution, and other issues. From the above reasons, energy harvesting technology plays an important role in improving the efficiency and life of electronic equipment. In order to explore the influence of the bimorph piezoelectric vibrator's structural parameters on the power generation capacity, this paper establishes a cantilever beam rectangular bimorph piezoelectric vibrator power generation model, derives the mathematical expression of the bimorph piezoelectric vibrator power generation, and determines the parameter factors that affect the power generation effect. Using MATLAB simulation analysis to obtain the influence relationship curve of system output voltage and structural parameters, the experiment tests the influence law of output voltage and thickness ratio, width-to-length ratio, and Young's modulus ratio; the test results are consistent with the theoretical analysis, verifying the theory and the correctness of the analysis. The results show that when the thickness ratio is 0.58 and the width-to-length ratio is 1 , the power generation effect of the piezoelectric vibrator is the best to reach $14.5 \mathrm{~V}$; the power generation capacity of the transducer is inversely proportional to Young's modulus ratio. This research provides a new idea for the popularization of integrated circuits, MEMS, and portable electronic devices.

\section{Introduction}

For wireless networks and embedded systems that are developing rapidly at present, the shortcomings of battery power supply are even more obvious. Therefore, how to power these low-energy-consumption electronic products has become an urgent problem to be solved. According to the different energy conversion mechanisms, there are three types of effective methods for obtaining electrical energy: electromagnetic [1,2], electrostatic [3], and piezoelectric $[4,5]$. Compared with other power generation principles, piezoelectric power generation has many advantages such as simple structure, no heat generation, no electromagnetic interference, no pollution, and easy realization of miniaturization and integration [6], and it can meet the energy supply of low energy consumption products. This demand has become a hot topic of current research.

Piezoelectric wafer transducers have the advantages of simple structure and high power generation efficiency, which have attracted the attention of the majority of researchers. In previous studies, scholars have mainly conducted research on the following aspect: Wang and Ma have studied ways to improve the power generation capacity of the transducer by arranging different segmented electrodes and mass blocks on the transducer [7, 8]; Tao, Zhang, and Jiangbo, through the theoretical analysis and testing of the wafer-type transducer to explore its power generation performance, researched whether increasing the resonance frequency band of the transducer power generation device can improve its power generation capacity [9-11]; Shan 
studied the power generation capacity of piezoelectric vibrators with different structures and analyzed the output characteristics of piezoelectric vibrators in different volumes [12]; Central South University and other institutions analyzed the energy capture of piezoelectric cantilever beams by studying linear piezoelectric theory and the relationship between the output power of the piezoelectric device and the physical parameters such as the piezoelectric structure, and it is concluded that, by optimizing the load impedance and changing the metal layer material, the energy harvesting efficiency of the piezoelectric energy harvester is effectively improved $[13,14]$; Gong Jun-Jie improved the power generation performance of the transducer by selecting appropriate structural parameter matching to make the natural frequency of the power generation device close to the external excitation frequency $[15,16]$. The above research only studies the structural properties of the piezoelectric transducer itself and ignores the influence of the ratio of the structural parameters of the substrate and the piezoelectric material on the output power of the transducer.

Different from the above research, Liu obtained the law of the output power of the transducer structure by analyzing the size parameters of the piezoelectric material [17]; Lei optimized the acquisition efficiency of the transducer by analyzing the relationship between the natural frequency and the size parameter [18]. But they still have not analyzed the influence of substrate size and material parameters on the transducer. This article innovatively establishes the theoretical model of the piezoelectric vibrator, analyzes the influence of the thickness ratio, width-to-length ratio, and Young's modulus ratio of the piezoelectric material to the substrate in the piezoelectric model on the power generation capacity, determining the optimal ratio of the structural parameters of the transducer and providing a new theoretical basis and technical reference for the field of wafertype energy conversion power generation.

\section{Structure and Mathematical Model}

According to research conducted by Hassan Elahi, in the process of comparing the rectangular PZT patch with the circular PZT patch, the voltage yield and harvest quality of the rectangular transducer are better than that of the circular PZT patch. Therefore, in order to maximize the power generation efficiency of the transducer, this paper adopts a rectangular structure transducer model [19].

The structure parameters and model of the bimorph transducer are shown in Figure 1. It adopts a cantilever support method and is mainly composed of a piezoelectric wafer and a metal substrate. The piezoelectric wafer is attached to the metal substrate on both sides, the transducer is bent and deformed under the action of the force, and then, bending stress is generated, and electric charge is generated on the surface of the piezoelectric ceramic to achieve the effect of power generation.

Suppose the total length of the bimorph transducer is $L$, the total width is $W$, and the total thickness is $h$. The subscripts $p$ and $m$ represent the piezoelectric wafer and the

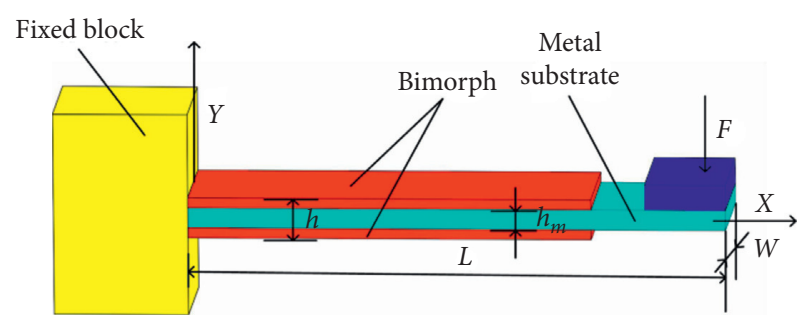

FIgURE 1: Structural parameters and model diagram of the piezoelectric transducer.

metal substrate, respectively. $\alpha=h_{m} / h$ represents the ratio of the thickness of the metal substrate $h_{m}$ to the total thickness of the piezoelectric vibrator $h$ :

$$
h_{p}=\frac{(1-\alpha) h}{2} \text {. }
$$

The relationship between the strain and stress of the piezoelectric wafer is

$$
\left\{\begin{array}{l}
T_{p}=E_{P}\left(S_{1}-g_{31} D_{3}\right), \\
E_{3}=-g_{31} T_{p}+\beta_{33}^{T} D_{3} .
\end{array}\right.
$$

In the above formula, $T_{p}$ and $S_{1}=-\rho z$, respectively, represent the stress and strain in the $x$ direction, $\rho$ represents the radius of curvature, $g_{31}$ represents the piezoelectric voltage constant, $E_{p}$ represents constant, $E_{p}$ represents Young's modulus, $E_{3}$ and $D_{3}$, respectively, represent the electric field strength and electric displacement, $\beta_{33}^{T}=1 / \varepsilon_{33}^{T}$ indicates the dielectric isolation rate, and $\varepsilon_{33}^{T}=1300 \varepsilon_{0}$ represents the dielectric constant.

The structure of the bimorph transducer is symmetrical, so the distance from the upper surface of the ceramic wafer to the center layer is

$$
z_{s}=\frac{h}{2}
$$

When a force is applied to the end of the transducer, its torque is

$$
M=n \int_{0}^{W} \int_{z_{s}-h_{p}}^{z_{s}} z T_{p} \mathrm{~d} y \mathrm{~d} z+\int_{0}^{W} \int_{-h+z_{s}}^{z_{s}-h_{p}} z T_{m} \mathrm{~d} y \mathrm{~d} z=(x-L) F .
$$

In the above formula, $n$ is the number of piezoelectric wafers, $T_{m}=E_{m} S_{1}$ represents the stress of the substrate in the $x$ direction, and $E_{m}$ is the Young's modulus of the substrate.

The radius of curvature is

$$
\rho=-\frac{3}{A E_{p} W h^{3}}\left[4(x-L) F+\left(1-\alpha^{2}\right) W h^{2} g_{31} E_{p} D_{3}\right],
$$

where $A=1-\alpha^{3}+\alpha^{3} \beta$, and $\beta=E_{m} / E_{p}$ is the ratio of Young's modulus of the metal substrate to the piezoelectric wafer.

Substituting formula (5) into formula (2) and integrating the voltage is 


$$
V=\int_{z_{s}-h_{p}}^{z_{s}} E_{3} \mathrm{~d} z=\frac{(1-\alpha)}{8 A W h}\left[12(1+\alpha) g_{31}(L-x) F+B W h^{2} \beta_{33}^{T} D_{3}\right] \text {, }
$$

where

$$
B=-3(1-\alpha)(1+\alpha)^{2} k_{31}^{2}+4 A\left(1+k_{31}^{2}\right), k_{31}^{2}=\frac{E_{p} g_{31}}{\beta_{33}^{T}} .
$$

Formula (6) as the electric displacement expression can be written as

$$
D_{3}=\frac{4}{(1-\alpha) \beta_{33}^{T} B W h^{2}}\left[3 g_{31}\left(1-\alpha^{2}\right)(x-L) F+2 A W h V\right] .
$$

Integrate the above formula to get the amount of charge generated by the transducer as

$$
Q=2 \int_{0}^{W} \int_{0}^{L} D_{3} \mathrm{~d} y \mathrm{~d} x=\frac{4 L^{2}}{\beta_{33}^{T} B h^{2}}\left[3(1+\alpha) g_{31} F+\frac{4 A W h V}{(1-\alpha) L}\right] .
$$

Since the applied electric field is 0 , the electricity generated by the single action is

$$
Q_{g}=\frac{12(1+\alpha) g_{31} L^{2}}{\beta_{33}^{T} B h^{2}} F,
$$

due to

$$
Q=\mathrm{CV} .
$$

From equation (9) and equation (11), the capacitance of the transducer is

$$
C=\frac{16 A W L}{(1-\alpha) \beta_{33}^{T} B h} .
$$

From equation (10) and equation (12), the voltage can be obtained as

$$
V_{g}=\frac{3\left(1-\alpha^{2}\right) L g_{31}}{4 W A h} F
$$

Set $\gamma=L / W$ as the width-to-length ratio of the transducer to obtain the voltage generated by the transducer under the action of external force:

$$
V_{g}=\frac{3\left(1-\alpha^{2}\right) \gamma g_{31}}{4\left(1-\alpha^{3}+\alpha^{3} \beta\right) h} F .
$$

\section{Simulation Analysis}

Select the specific parameters of the piezoelectric wafer transducer, as shown in Table 1. Through the above theoretical analysis, it is concluded that the power generation of the transducer is related to the thickness ratio, width-to-length ratio, and Young's modulus ratio. The external driving force is $1 \mathrm{~N}$, the excitation amplitude is $2.0 \mathrm{~mm}$, and the MATLAB software is used for simulation analysis.

The influence curve of the thickness ratio on the power generation of the transducer is shown in Figure 2. It can be seen from the simulation curve that the power generation increases first and then decreases as the thickness ratio of the transducer increases. When the thickness ratio is 0.54 , the output voltage reaches the maximum.

The influence curve of the width-to-length ratio on the power generation of the transducer is shown in Figure 3. It can be seen from the simulation graph that when the interval is $0-1$, the greater the width-to-length ratio and the stronger the power generation capacity of the transducer. However, when the width-to-length ratio exceeds 1 , as the width-to-length ratio increases, the voltage output capability weakens.

Different substrate materials have different Young's modulus, resulting in different power generation capabilities. The curve of the influence of Young's modulus ratio on the power generation of the transducer is shown in Figure 4 . It can be seen from the simulation graph that the output power of the transducer is inversely proportional to the ratio of Young's modulus.

\section{Comparative Analysis of Theory and Experiment}

4.1. Test Device. According to the research on energy harvesting by Hassan et al., this experimental test uses an energy harvesting architecture [20]; the system test device is mainly composed of an oscilloscope, a power amplifier, a vibration exciter, a bimorph rectangular transducer, a support frame, etc. The specific physical device diagram is shown in Figure 5. The vibration exciter is used to excite the piezoelectric transducer to produce vibration, and the excitation amplitude is set to $2.0 \mathrm{~mm}$. Change the thickness, size, and material properties of the piezoelectric wafer and the metal substrate, and test the influence of the thickness ratio, the width-to-length ratio, and Young's modulus ratio on the power generation effect.

4.2. The Influence of Thickness Ratio on Output Voltage. The influence curve of the transducer thickness ratio on the output voltage is shown in Figure 6. It can be seen from the figure that the output voltage of the transducer first increases and then decreases with the thickness ratio. The theoretical value is consistent with the test value curve trend. The theoretical value peaks at a thickness ratio of 0.52 , while the 
TABle 1: Transducer parameters.

\begin{tabular}{lcccc}
\hline Material & Piezoelectric constant $g_{31} \times 10^{-3}$ & Young's modulus $E_{p}(\mathrm{GPa})$ & Poisson's ratio & $\begin{array}{r}\text { Vacuum dielectric } \\
\text { constant } \varepsilon_{0} \times 10^{-12}\end{array}$ \\
\hline PZT-5H & 9.11 & 60.6 & 0.34 & 8.85 \\
$\mathrm{Cu}$ & - & 115 & 0.31 & - \\
\hline
\end{tabular}

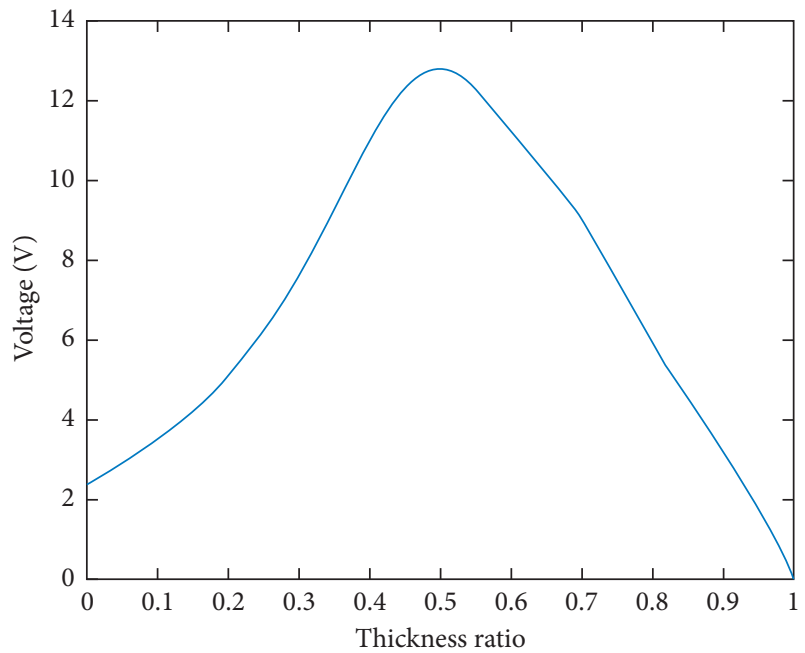

FIgURE 2: The influence curve of the thickness ratio on the power generation of the transducer.

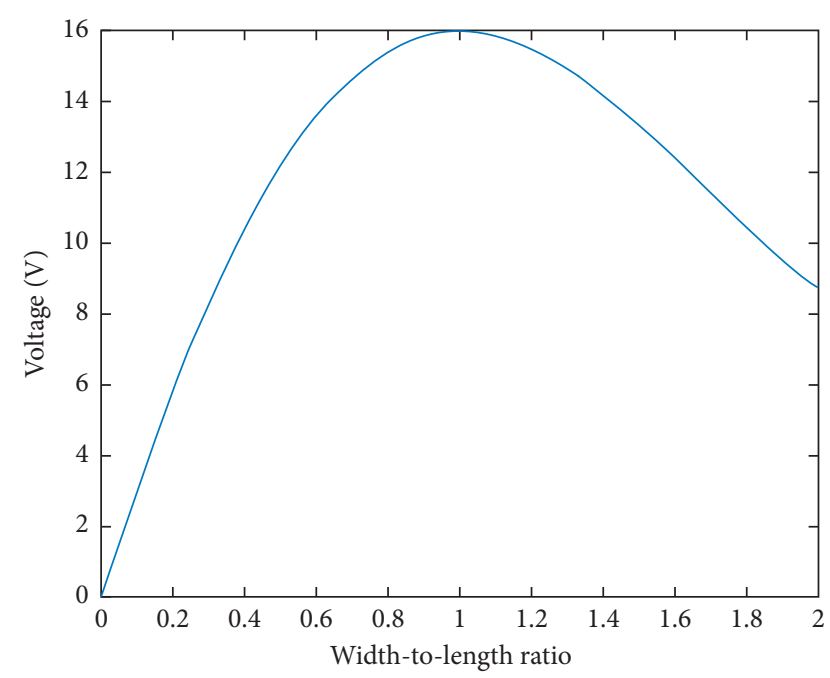

FIgURE 3: The influence curve of the width-to-length ratio on the power generation of the transducer.

actual value peaks at the thickness ratio of 0.58 . When the thickness ratio is 0.58 , the output voltage is the maximum, and the output voltage is $12.3 \mathrm{~V}$ at this time.

4.3. The Effect of Width-to-Length Ratio on Output Voltage. The influence curve of the width-to-length ratio of the transducer on the output voltage is shown in Figure 7. It can be seen from the figure that the theoretical value is consistent

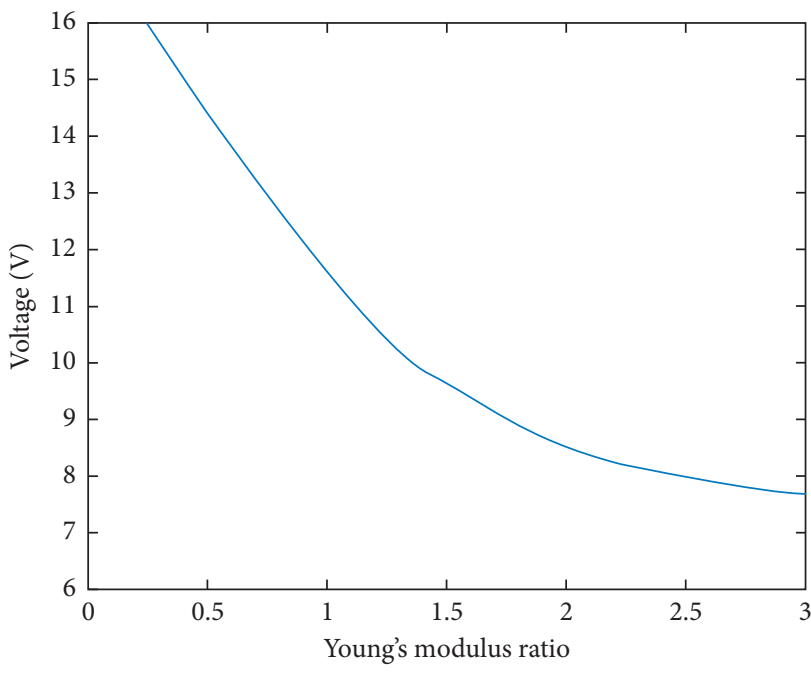

FIgURE 4: The influence curve of Young's modulus ratio on the power generation of the transducer.

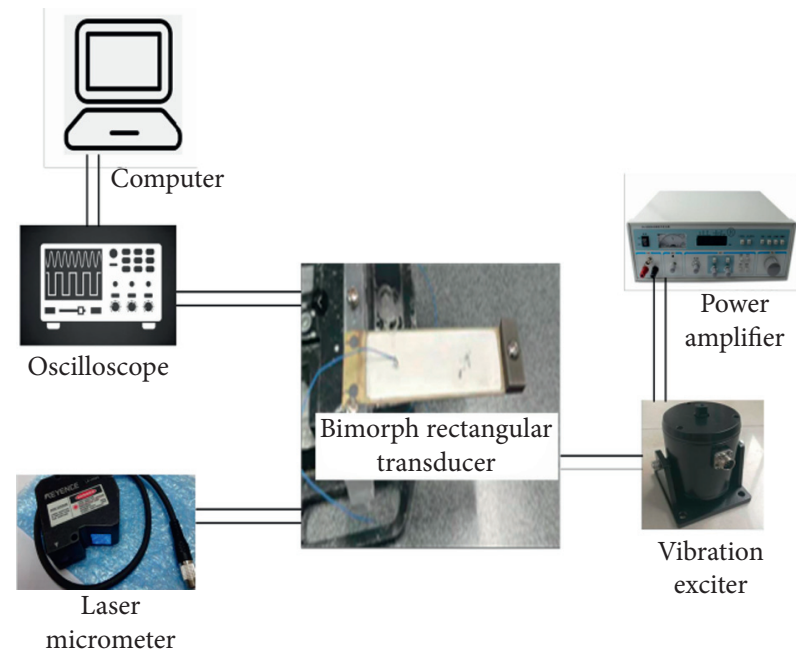

FIgure 5: Test device.

with the trend of the test value curve. The voltage output of the transducer is proportional to the aspect ratio in the $0-1$ interval. The larger the aspect ratio, the higher the output voltage. When the aspect ratio is close to 1 , the output voltage reaches the maximum value of $14.5 \mathrm{~V}$, and the electric energy output is the best; when the width-to-length ratio interval exceeds 1 , the output voltage capability decreases. 


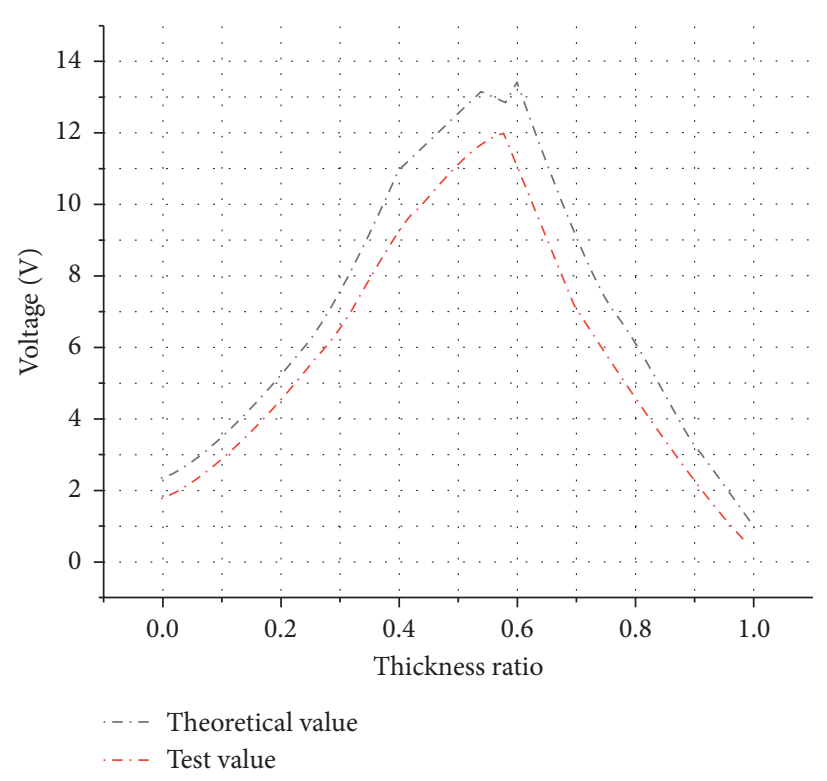

Figure 6: The relationship between thickness ratio and output voltage.

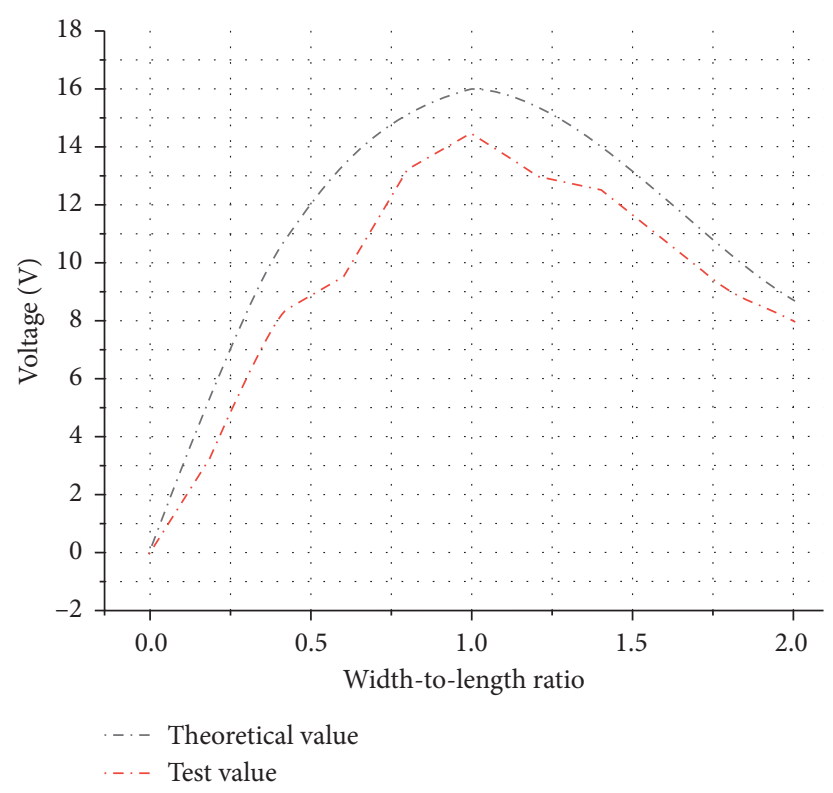

Figure 7: The relationship between the width-to-length ratio and the output voltage.

4.4. The Influence of Young's Modulus Ratio on the Output Voltage. The influence curve of transducer Young's modulus ratio on the output voltage is shown in Figure 8. It can be seen from the figure that the theoretical value is consistent with the trend of the test value curve. The output voltage gradually decreases as Young's modulus ratio increases, and the output voltage and Young's modulus ratio show an inverse relationship. 


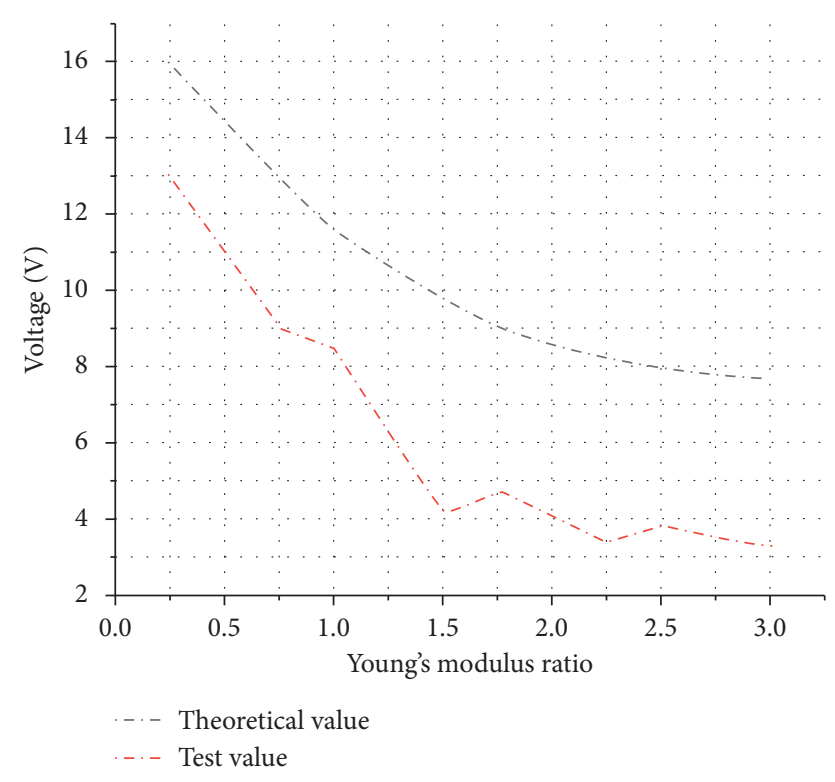

FIgURE 8: The relationship curve between Young's modulus ratio and output voltage.

\section{Conclusion}

(1) The power generation model of the cantilever beam rectangular piezoelectric vibrator was established, and the power generation model was simulated and analyzed by MATLAB. Analyze the influence of structural parameters on the piezoelectric power generation of the cantilever through experimental tests, and the theoretical analysis is consistent with the experimental test results.

(2) The output voltage of the cantilever piezoelectric vibrator is proportional to the excitation amplitude, and the output voltage increases first and then decreases with the thickness ratio, width-to-length ratio, and Young's modulus ratio. When the thickness ratio is 0.58 and the width-to-length ratio is 1 , the output voltage effect of the piezoelectric vibrator is the best.

(3) The power generation capacity of the transducer is inversely proportional to the ratio of Young's modulus of the piezoelectric wafer to the metal substrate.

The above conclusions provide a new idea for considering the size and material parameter ratio optimization of the piezoelectric energy harvester's substrate and piezoelectric material. It has also further promoted the optimization of integrated circuits and the popularization of MEMS and portable electronic equipment applications.

\section{Data Availability}

The data used to support the findings of this study are available from the corresponding author upon reasonable request.

\section{Conflicts of Interest}

The authors declare that there are no conflicts of interest regarding the publication of this paper.

\section{Acknowledgments}

This work was supported by the National Natural Science Youth Foundation of China under Grant nos. 51705031 and 51875234, Science and Technology Development Plan of Jilin Province of China under Grant no. 20200201063JC, and Scientific Research of Education Department of Jilin Province of China under Grant no. JJKH20210608KJ.

\section{References}

[1] C. Liu, R. W. Chen, Y. X. Zhang, L. P. Wang, and J. C. Qin, “A novel fully floating three-body system for direct-drive wave energy converter," International Journal of Applied Electromagnetics and Mechanics, vol. 65, no. 5, pp. 803-818, 2020.

[2] J. Liu, H. W. Sun, Y. T. Li, W. L. Fang, and S. B. Niu, "An improved power system transient stability prediction model based on mRMR feature selection and WTA ensemble learning," Applied Sciences, vol. 10, no. 7, Article ID 2255, 2020.

[3] G. Chen, L. H. Tang, Z. S. Yang, K. Tao, and Z. B. Yun, “An electret-based thermoacoustic-electrostatic power generator," Energy Weekly News, vol. 44, pp. 1-8, 2019.

[4] J. Luo, Z. Cao, M. Yuan, Y. Liang, X. Xu, and M. Li, "Fabrication and characterization of miniature nonlinear piezoelectric harvester applied for low frequency and weak vibration," Results in Physics, vol. 11, pp. 237-242, 2018.

[5] R. Harada, N. Y. Iwamoto, S. H. Kweon, T. Umegaki, and I. Kanno, "Finger flexion power generators made of piezoelectric lead zirconate titanate thin films on stainless steel foils," Sensors and Actuators, A: Physical, vol. 322, Article ID 112617, 2021.

[6] B. Wu, S. J. E, Z. G. Yang, and G. M. Chen, "Development and application of piezoelectric actuation and control," Chinese Journal of Mechanical Engineering, vol. 39, no. 10, pp. 79-85, 2003.

[7] H. Y. Wang, X. B. Shan, T. Xie, and J. B. Yuan, "Effect of electrode configuration on electricitygenerating capacity of a piezoelectric cantilever," Journal of Vibration and Shock, vol. 30, no. 12, pp. 129-134, 2011.

[8] X. Q. Ma, L. J. Gong, Q. S. Pan, Y. X. Wu, C. Liu, and Y. Wu, "Analysis of the influence of mass block position on the power generation performance of piezoelectric cantilever beam," Piezoelectrics and Acoustooptics, vol. 40, no. 5, pp. 789-792+798, 2008.

[9] J. X. Tao, N. V. Viet, A. Carpinteri, and Q. Wang, "Energy harvesting from wind by a piezoelectric harvester," Engineering Structures, vol. 133, pp. 74-80, 2017.

[10] J. Zhang, Z. Fang, C. Shu, J. Zhang, Q. Zhang, and C. Li, “A rotational piezoelectric energy harvester for efficient wind energy harvesting," Sensors and Actuators A: Physical, vol. 262, pp. 123-129, 2017.

[11] J. B. Yuan, T. Xie, W. S. Chen, and N. Xiao, "Experimental study on electricity generating capacity for a piezoelectric cantilever," Journal of Vibration and Shock, vol. 28, no. 7, pp. 69-72+213, 2009.

[12] X. B. Shan, J. B. Yuan, T. Xie, and W. S. Chen, "Modeling and test of piezoelectric cantilever generators with different 
shapes," Journal of Vibration and Shock, vol. 29, no. 4, pp. 177-180+239, 2010.

[13] S. N. Jiang, S. H. Guo, and X. F. Li, "Performance analysis for a unimorph cantilever piezoelectric harvester," Journal of Vibration and Shock, vol. 31, no. 19, pp. 90-94, 2012.

[14] X. Z. Wu, S. Y. Yan, Z. Q. Gao, J. Han, and G. T. Jiao, "Analysis of influence of cantilever beam substrate on output response of piezoelectric energy harvester," Piezoelectrics and Acoustooptics, vol. 42, no. 5, pp. 686-691, 2020.

[15] J. J. Gong, Y. Y. Xu, Z. L. Ruan, and Y. X. Bian, "Simulation on generating capacity for energy harvesting device with piezoelectric bimorph cantilever," Journal of Vibration, Measurement \& Diagnosis, vol. 34, no. 4, pp. 658-663+776, 2014.

[16] A. Abdelkefi, N. Barsallo, L. Tang, Y. Yang, and M. R. Hajj, "Modeling, validation, and performance of low-frequency piezoelectric energy Harvesters," Journal of Intelligent Material Systems and Structures, vol. 25, no. 12, pp. 1429-1444, 2014.

[17] X. J. Liu and R. W. Chen, "Analysis of load voltage and output power for rainbow shape piezoelectric monomorph energy transferring elements," Acta Aeronautica et Astronautica Sinica, vol. 32, no. 3, pp. 561-570, 2011.

[18] X. H. Lei, Y. H. Wu, S. S. Zhu, and X. P. Qian, “Theoretical and experimental study of piezoelectric energy acquisition efficiency with cantilever beam," Transducer and Microsystem Technologies, vol. 39, no. 11, pp. 18-20+24, 2020.

[19] H. Elahi, "The investigation on structural health monitoring of aerospace structures via piezoelectric aeroelastic energy harvesting," Microsystem Technologies, vol. 27, pp. 1-9, 2020.

[20] E. Hassan, M. Khushboo, E. Marco, A. Sofiane, and G. Paolo, "Energy harvesting towards self-powered IoT devices," $\mathrm{Nu}$ trients, vol. 13, no. 21, p. 5528, 2020. 